\title{
Radiation Field
}

National Cancer Institute

\section{Source}

National Cancer Institute. Radiation Field. NCI Thesaurus. Code C54648.

The area of the body exposed to radiation during radiation therapy. 